AIJIA ZHANG, Ph.D. ${ }^{1}$

E-mail: zhangaijiaseu@foxmail.com

TIEZHU LI, Ph.D. ${ }^{1}$

(Corresponding author)

E-mail: litiezhu@seu.edu.cn

RAN TU, Ph.D. ${ }^{1}$

E-mail: turanjade@outlook.com

CHANGYIN DONG, Ph.D. ${ }^{1}$

E-mail: dongcy@seu.edu.cn

HAIBO CHEN, Ph.D. ${ }^{2}$

E-mail: H.Chen@its.leeds.ac.uk

JIANBING GAO, Ph.D. ${ }^{2}$

E-mail: redonggaojianbing@163.com

YE LIU, Ph.D. ${ }^{2}$

E-mail:Y.Liu8@leeds.ac.uk

${ }^{1}$ School of Transportation, Southeast University

Nanjing, China

${ }^{2}$ Institute for Transport Studies

University of Leeds

Leeds, UK
Intelligent Transport Systems (ITS)

Original Scientific Paper

Submitted: 5 Nov. 2020

Accepted: 12 Apr. 2021

DOI: $10.7307 /$ ptt.v33i4.3730

\title{
THE EFFECT OF NONLINEAR CHARGING FUNCTION AND LINE CHANGE CONSTRAINTS ON ELECTRIC BUS SCHEDULING
}

\begin{abstract}
The recharging plans are a key component of the electric bus schedule. Since the real-world charging function of electric vehicles follows a nonlinear relationship with the charging duration, it is challenging to accurately estimate the charging time. To provide a feasible bus schedule given the nonlinear charging function, this paper proposes a mixed integer programming model with a piecewise linear charging approximation and multi-depot and multi-vehicle type scheduling. The objective of the model is to minimise the total cost of the schedule, which includes the vehicle purchasing cost and operation cost. From a practical point of view, the number of line changes of each bus is also taken as one of the constraints in the optimisation. An improved heuristic algorithm is then proposed to find high-quality solutions of the problem with an efficient computation. Finally, a real-world dataset is used for the case study. The results of using different charging functions indicate a large deviation between the linear charging function and the piecewise linear approximation, which can effectively avoid the infeasible bus schedules. Moreover, the experiments show that the proposed line change constraints can be an effective control method for transit operators.
\end{abstract}

\section{KEYWORDS}

electric bus scheduling problem; nonlinear charging function; line change constraints; mixed integer optimisation; heuristic algorithm.

\section{INTRODUCTION}

Due to the environmental benefits of electric vehicles, vehicle electrification is now regarded as the main decarbonisation pathway, especially for public transit buses. Various bus electrification plans have been implemented under government incentives, such as the TIGER program in the United States, the Green Bus Fund Program in the UK, the Electric Mobility program in Germany, and the Ten Cities and Thousand Vehicles Program in China [1]. With this trend, electric buses will have a large market share in the future public transit system. The adoption of electric buses requires comprehensive plans for facilities and operation strategies. In the bus operational planning, the scheduling is one of the key parts, which focuses on the bus type selection and the trip timetable design in a cost-efficient way.

The electric bus scheduling problem is an extension of the traditional vehicle scheduling problem (VSP). VSP refers to the problem of determining the optimal assignment of vehicles to carry out all the trips in a given transit timetable. The objective is to find a schedule that can complete all the trips with the minimum number of vehicles or the minimum total cost [2]. An important branch among different types of the VSP is the multi-depot vehicle scheduling problem (MDVSP), in which multiple 
depots exist in the network and vehicles are forced to return to their departure depots after performing their scheduled trips. The MDVSP is proved as an NP-hard problem [3] and is usually solved by the heuristic approach to achieve an acceptable computation time. Another important VSP variant is the multi-vehicle type scheduling problem (MVTVSP), which uses multiple available vehicle types to complete the timetable trips. Bunte and Kliewer [4] reviewed the modelling approaches for different kinds of VSPs and gives the basic ideas of solution approaches.

For the bus scheduling problem, to increase the vehicle utilisation rate and reduce the operation cost, bus companies usually allow buses to shift among different lines for more trips, which is also called "mixed-line" strategy. Kliewer, Mellouli [5] adopted this strategy and proposed a model based on the time-space network theory to optimise the bus scheduling with multi-depots. However, this strategy is not always cost-efficient. A large number of line changes creates more deadhead mileages, increases energy consumption, and raises the risk of delays. It also possibly leads to inconveniences for the bus and crew management, especially in a large transit network. [6] extended their research to find a low number of different lines per bus rotation when the schedules are cost-minimal. However, few studies have been conducted to investigate the bus scheduling problem with limited line changes.

With the fast-growing market share of electric vehicles, research studies on the electric vehicle scheduling problem (EVSP) have emerged. Different from the conventional fuel vehicle scheduling problem, the electric vehicle scheduling problem needs to consider the driving range and recharging constraints. To design an appropriate recharging plan for the fleet, it is essential to accurately estimate the charging time of the vehicles. The linear charging function or the fixed time estimation method is often applied in the research. Chao and Xiaohong [7] proposed a single depot scheduling model with a non-dominated sorting genetic algorithm to optimise the battery swapping buses schedule. Li [8] investigated the single depot bus scheduling problem considering battery swapping and fast charging vehicles. The column-generation-based algorithm was developed to solve the problem. Wen, Linde [9] developed a mixed-integer programming model for EVSP with multi-depots (multi depot electric vehicle scheduling problem, MDEVSP), and the partial charging policy was adopted. The model was solved by an adaptive large neighbourhood search algorithm. Tang, Lin [10] proposed a robust scheduling model within a branch-and-price solution framework to handle the stochastic traffic conditions. Some other research focused on the EVSP with heterogeneous fleet (multi-vehicle type electric vehicle scheduling problem, MVTEVSP), which can provide more practical suggestions for the fleet management. Rogge, van der Hurk [11] studied the MVTEVSP with the optimisation of charging infrastructure. A grouping genetic algorithm with mixed-integer charging infrastructure optimisation was developed to minimise the total cost of ownership. Li, Lo [12] studied the bus scheduling problem considering a mixed fleet composed of both electric and conventional fuel buses. The problem is formulated as an integer linear program which could be solved by mathematical programming solver. Rinaldi, Picarelli [13] developed a mixed integer linear program to address the mixed electric bus fleet scheduling problem, coupled with an ad-hoc decomposition scheme to enhance the scalability of the model.

However, the state of charge during the charging process usually shows a nonlinear relationship with the charging time due to the change of the terminal voltage and current [14]. Therefore, it means that if a vehicle starts charging at different states of charge, the charging time required for the same amount of charged electrical energy can be varied. Although the linear charging function or fixed time estimation method can simplify the modelling and solving methods of the EVSPs, it may lead to a wrong estimated charging time compared to the actual charging duration. The simplification can cause operational problems, especially for public transit buses. Overestimating the charging time results in a waste of bus operating time, while the underestimation leads to insufficient state of charge for a complete bus service. To solve this problem, Montoya, Guéret [15] studied the electric vehicle routing problem with nonlinear function, the piecewise linear approximation approach is used to accurately approximate the charging function of the vehicle. Froger, Mendoza [16] proposed an improved pathbased model for the problem to avoid replicating the charging stations nodes and thus reduced the computational time. However, models and algorithms 
proposed in previous studies only focused on vehicle routing problems, whereas the multi-depot and multi-vehicle type conditions are rarely considered.

To fill these gaps, this paper investigates the electric bus scheduling problem with a nonlinear charging function. From a practical perspective, the multi-depot and multi-vehicle type conditions are considered in the optimisation of the total cost of the schedule (multi-depot and multi-vehicle type electric vehicle scheduling problem with nonlinear charging function). Besides, to improve the mixedline strategy in bus scheduling, constrains are set to restrict the upper bound number of line changes for each vehicle in the fleet. The transit operators can control the number of bus line changes while seeking a low cost. An adaptive large neighbourhood search heuristic algorithm (ALNS) with improved mechanisms is developed to solve the problem more efficiently. This methodology can provide effective suggestions for the electrification of the public transportation.

The paper is organised as follows. Section 2 presents the developed mathematical formulation of the optimisation problem and the heuristic algorithm for solving the problem. A case study with the real-world operation data is conducted by applying the proposed optimisation algorithm. The results are analysed in Section 3. Finally, a summary of the results and future studies is provided in Section 4.

\section{METHODOLOGY}

\subsection{Optimisation problem}

The optimisation problem in this paper can be defined as follows: Let $D=\left\{\beta_{1}, \ldots, \beta_{n}\right\}$ be the set of depots $\beta$ at which the buses can recharge their battery and park. Let $K$ be the set of electric bus types. Each bus type $k \in K$ has the battery capacity $E^{k}$, energy consumption rate $\tau_{k}$, and purchase $\operatorname{cost} c_{k}$.
Let $S$ be the set of timetable trips, which contains the trips of several bus lines. When the bus of type $k$ performs a trip $s$, the time it takes is denoted as $t_{s}$ and the electrical energy it consumes is denoted as $e_{s}^{k}$. During the operation, the bus is allowed to shift from one bus line to another, but no more than the maximum number of $\theta$ times. The state of charge of each bus in operation must be kept above the safe driving level. If the bus needs to be recharged during operation, it needs to return to its departure depot for recharging after finishing the current trip. The set $F=\left\{f_{1}, \ldots, f_{r}\right\}$ denotes all the recharging trips $f$. In a single charge, the bus can be recharged for any amount of energy until the battery is fully charged. Once the bus finished its schedule, it needs to return to its departure depot.

The objective of the optimisation is to minimise the operating cost of the bus fleet by determining the type of buses, the number of each type, the scheduling and recharging plan of all buses. A solution of the problem is feasible if (1) all timetable trips are performed exactly once, (2) the schedule does not violate the trips' time window, (3) the bus service will not be broken down due to insufficient state of charge, (4) the number of line changes of each bus does not exceed the limitation.

\subsection{Modelling the charging function}

As is mentioned in the Introduction, the real-world charging functions are usually nonlinear [14]. A typical two-phase charging curve is shown in Figure 1. This charging procedure is widely used in practical operations of lithium-ion battery, which is currently the most widely used battery type for electric vehicles [17]. The vehicle is charged at a constant current (CC phase) in the first phase, and state of charge (SOC) increases linearly. In the second phase, the vehicle is charged at a constant

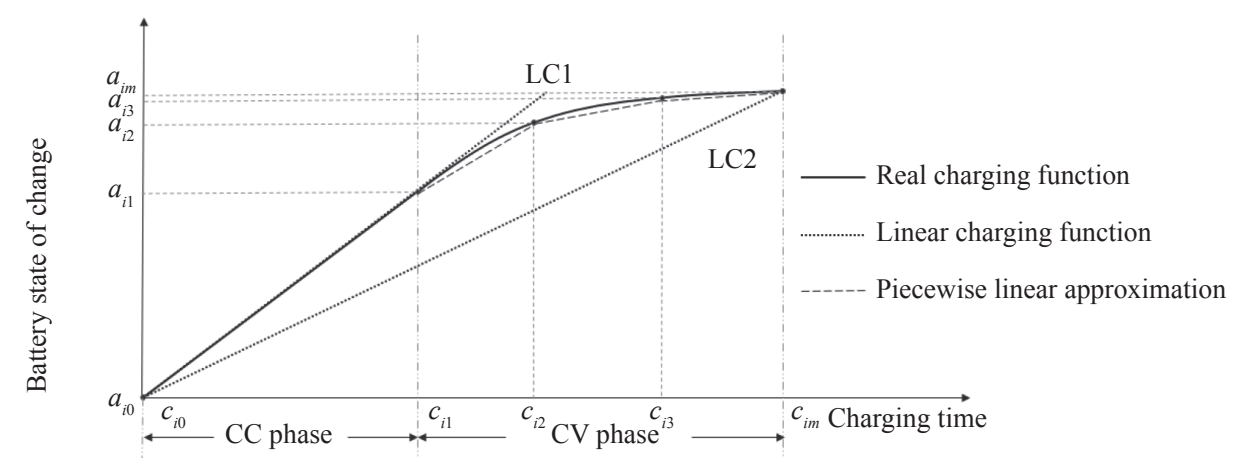

Figure 1 - An illustration of linear vs. real charging function and the piecewise linear approximation approach Source: Hõimoja, Rufer [18]; Montoya, Guéret [15] 
voltage (CV phase) and decreasing current, and the charging curve is concave. Two common linear approximating functions mentioned in [15] are also shown in the figure. The first approximation (LC1) directly uses the charging rate in the $\mathrm{CC}$ phase as the charging rate of the whole process. The second approximation (LC2) is more conservative, which calculates the average charging rate of the whole process. Both linear functions have a clear deviation from the actual charging function, which may result in overestimation or underestimation of the actual charging time, thus leading to infeasible schedules.

To avoid the error in charging time estimation, the piecewise linear approximation approach is used to model the real-world charging function. For a bus type $k$, a specific piecewise linear charging function $\phi^{k}$ is provided. Let $B^{k}=\left\{0, \ldots, m^{k}\right\}$ denote the breakpoints of the function, where $m$ is the number of the breakpoints. Let $c_{f b}^{k}$ and $a_{f b}^{k}$ represent the charging time and state of charge corresponding to each breakpoint on the piecewise linear function, where $b$ and $f$ represent the breakpoint and recharging trip, respectively. The dashed line in Figure 1 shows a piecewise linear approximation function consisted of 4 secant lines and 5 breakpoints. Compared with the two linear functions, the piecewise linear approximation has a better fit with the real-world charging curve. In the piecewise linear approximation, the number of breakpoints is user-defined. The approximation becomes more accurate as more breakpoints are used [19].

\subsection{Mathematical formulation}

The model of the problem is defined on a directed multigraph $G=(V, A) . V=o^{\beta} \cup d^{\beta} \cup S \cup F$ represent all nodes in the graph. Nodes $o^{\beta}$ and $d^{\beta}$ denote the start and end nodes for the depot $\beta \in D$, respectively. $A$ represents all feasible arcs, representing all deadhead trips in the schedule. For a node $i \in V$ representing a trip, the start time is denoted as $z_{i}$, and the travel time is denoted as $t_{i}$. For an $\operatorname{arc} a=(i, j), a \in A$, the travel time, distance, and the energy consumption of the arc are denoted by $t_{i j}, d_{i j}$, and $e_{i j}^{k}$. For simplification, in the model, we use the notation $t_{a}, d_{a}$, and $e_{a}^{k}$ instead of $t_{i j}, d_{i j}$, and $e_{i j}^{k}$. When the bus performs a service trip or a deadhead trip, the unit electricity cost is $c_{e}$ and unit time cost is $c_{e}$. For a recharging trip $f \in F$, let $q_{f}^{k}$ and $\overline{q_{f}^{k}}$ denote the state of charge (according to the piecewise linear approximation of the charging function) of the bus of type $k$ at the beginning and end of recharging trips, respectively, $c_{f}^{k}$ and $\overline{c_{f}^{k}}$ being the time when the bus starts and finishes charging, respectively. Let the binary variables $w_{f b}^{k}$ and $\overline{w_{f b}^{k}}$ equal $1, b \in B^{k}\{\{0\}$, if the state of charge of the bus is between the breakpoints $a^{k}$ $f, b-1$ and $a_{f b}^{k}$ of the piecewise linear approximation. Besides, the continuous variables $\lambda_{f b}^{k}$ and $\overline{\lambda_{f b}^{k}}$ are the coefficients associated with the breakpoint $\left(c_{f b}^{k}, a_{f b}^{k}\right)$ in the piecewise linear approximation, when the bus starts and finishes charging, respectively. It is worth mentioning that theoretically buses can be recharged after any service trip; in the directed graph $G$, each service trip node is followed by a possible recharging node. The node would be connected to two service trip nodes if it is used, otherwise it becomes an isolated node, which is not connected into the graph. Set $F$ contains both used and isolated recharging nodes. Let $l_{i}^{\beta k}$ denote the number of line changes of the bus pass node $i$, whose bus type is $k$ and departing from depot $\beta$. Since all nodes except the departure and arrival nodes can only be passed by one bus once, this parameter can track the number of line changes of each bus.

The binary variable $x_{a}^{\beta k}$ represents the connections in the graph, where $a=(i, j)$ is the arc of any two nodes. $x_{a}^{\beta k}$ is 1 if a bus of type $k$ from $\operatorname{depot} \beta$ is operating on arc $a=(i, j)$. For simplicity, let $\Delta^{+}(i)$ and $\Delta^{-}(i)$ denote all the arcs starting or ending at node $i$, respectively. Figure 2 shows an example of the directed graph $G$ with 2 depots and 8 service trips in total.

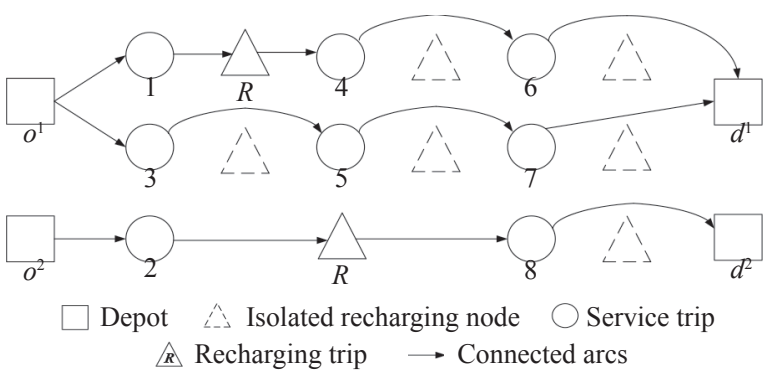

Figure 2-An illustration of the directed graph

The mathematical model can be formulated as follows:

Objective function

$$
\begin{aligned}
& \sum_{k \in K} \sum_{\beta \in D} \sum_{a \in \Delta^{+}\left(o^{\beta}\right)} c_{k} x_{a}^{\beta k} \\
& +\sum_{k \in K} \sum_{\beta \in D} \sum_{a=(i, j) \in A, i \in S \cup o^{\beta}, j \in V, i \neq j}\left(e_{i}^{k}+e_{a}^{k}\right) c_{e} x_{a}^{\beta k} \\
& +\sum_{k \in K} \sum_{\beta \in D}\left(t_{i}+t_{a}\right) c_{t} x_{a}^{\beta k}
\end{aligned}
$$




$$
\begin{aligned}
& \sum_{k \in K} \sum_{\beta \in D} \sum_{a \in \Delta^{+}(i)} x_{a}^{\beta k}=1, \quad \forall i \in S \\
& \sum_{k \in K} \sum_{\beta \in D} \sum_{a \in \Delta^{+}(f)} x_{a}^{\beta k} \leq 1, \quad \forall f \in F, \forall \beta \in D \\
& \sum_{k \in K} \sum_{\beta^{\prime} \in D \backslash\{\beta\}} \sum_{a \in \Delta^{+}\left(o^{\beta}\right)} x_{a}^{\beta^{\prime} k}=0, \quad \forall \beta \in D \\
& \sum_{k \in K} \sum_{\beta^{\prime} \in D \backslash\{\beta\}} \sum_{a \in \Delta^{-}\left(d^{\beta}\right)} x_{a}^{\beta^{\prime} k}=0, \quad \forall \beta \in D \\
& \sum_{a \in \Delta^{+}(i)} x_{a}^{\beta k}-\sum_{a \in \Delta^{-}(i)} x_{a}^{\beta k}=0 \quad \forall i \in S \cup F, \forall k \in K, \forall \beta \in D \\
& \underline{q_{f}^{k}} \leq \overline{q_{f}^{k}} \quad \forall f \in F, \forall k \in K \\
& \underline{q_{f}^{k}}=\sum_{b \in B^{k}} \underline{\lambda_{f b}^{k}} a_{f b}^{k} \quad \forall f \in F, \forall k \in K
\end{aligned}
$$

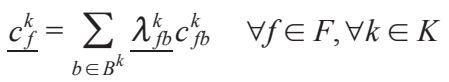

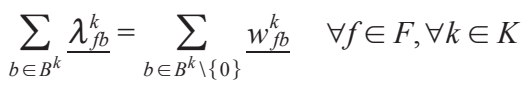

$$
\begin{aligned}
& \sum_{b \in B^{k} \backslash\{0\}} \frac{w_{f b}^{k}=}{k \in K} \sum_{\beta \in D} x_{a}^{\beta k} \\
& \forall f \in F, \forall k \in K, \forall a=(f, j) \in A \\
& \underline{\lambda_{f 0}^{k}} \leq \underline{w_{f 1}^{k}} \quad \forall f \in F, \forall k \in K \\
& \underline{\lambda_{f b}^{k}} \leq \underline{w_{f b}^{k}}+\underline{w_{f b+1}^{k}} \quad \forall f \in F, \forall k \in K, \forall b \in B^{k} \backslash\left\{0, m^{k}\right\} \\
& \underline{\lambda_{f m^{k}}^{k}} \leq \underline{w_{f m^{k}}^{k}} \quad \forall f \in F, \forall k \in K \\
& \overline{q_{f}^{k}}=\sum_{b \in B^{k}} \overline{\lambda_{f b}^{k}} a_{f b}^{k} \quad \forall f \in F, \forall k \in K \\
& \overline{c_{f}^{k}}=\sum_{b \in B^{k}} \overline{\lambda_{f b}^{k}} c_{f b}^{k} \quad \forall f \in F, \forall k \in K \\
& \sum_{b \in B^{k}} \overline{\lambda_{f b}^{k}}=\sum_{b \in B^{k} \backslash\{0\}} \overline{w_{f b}^{k}} \quad \forall f \in F, \forall k \in K \\
& \sum_{b \in B^{k} \backslash\{0\}} \overline{w_{f b}^{k}}=\sum_{k \in K} \sum_{\beta \in D} x_{a}^{\beta k} \\
& \forall f \in F, \forall k \in K, \forall a=(f, j) \in A \\
& \overline{\lambda_{f 0}^{k}} \leq \overline{w_{f 1}^{k}} \quad \forall f \in F, \forall k \in K \\
& \overline{\lambda_{f b}^{k}} \leq \overline{w_{f b}^{k}}+\overline{w_{f b+1}^{k}} \quad \forall f \in F, \forall k \in K, \forall b \in B^{k} \backslash\left\{0, m^{k}\right\} \\
& \overline{\lambda_{f m^{k}}^{k}} \leq \overline{w_{f m^{k}}^{k}} \quad \forall f \in F, \forall k \in K \\
& z_{i}+t_{i}+t_{a}-M\left(1-\sum_{k \in K} \sum_{\beta \in D} x_{a}^{\beta k}\right) \leq z_{j} \\
& \forall i \in S, \forall a=(i, j) \in \Delta^{+}(i) \\
& z_{f}+\left(\overline{c_{f}^{k}}-\underline{c_{f}^{k}}\right)+t_{a}-M\left(1-\sum_{k \in K} \sum_{\beta \in D} x_{a}^{\beta k}\right) \leq z_{j} \\
& \forall f \in F, \forall a=(f, j) \in \Delta^{+}(f) \\
& y_{i}^{k}-e_{i}^{k}-\tau_{k} \cdot d_{a}+M\left(1-\sum_{k \in K} \sum_{\beta \in D} x_{a}^{\beta k}\right) \geq y_{j}^{k} \\
& \forall i \in S \cup o^{\beta}, \forall a=(i, j) \in \Delta^{+}(i)
\end{aligned}
$$

Objective 1 minimises the total cost of the schedule, which is split into three components. The first component calculates the purchase cost of all buses. The second component is the electricity consumption cost in the schedule, while the last component is the time-dependent cost in the schedule. Constraint 2 enforces that each service trip is covered exactly once. Constraint 3 ensures that each possible recharging trip can be performed at most once. Constraints 4 and 5 ensure that the number of buses departed and returned from each depot is the same, and constraint 6 is the flow conservation constraint. Constraint 7 ensures that the state of charge after charging is greater than that before charging. Constraints 8 and 9 denote the state of charge and charging time when the bus starts charging according to the piecewise linear approximation. Constraint 10 couples the coefficient related to the breakpoint with the binary variable representing the state of charge. Constraint 11 couples two kinds of binary variables. Constraints 12-14 define the relationship between the coefficient and the binary variable. Similarly, Constraints 15-21 define the state of charge and charging time when a bus finishes charging. Constraint 22 ensures that the two nodes continuously visited by the same bus do not violate the time window of any trips, where $M$ is a sufficiently large positive number. Likewise, constraint 23 ensures that the bus has enough time to arrive at 
the start location of the next trip after recharging. Similarly, constraints 24 and 25 ensure that the bus has enough energy to reach the start location of the next trip after performing the previous service trip or recharging trip. Constraint 26 ensures that the number of line changes of all buses is 0 when departing from the depot for the first time. Constraints 27 and 28 track the number of line changes of the bus. Constraint 29 ensures that the number of times the bus changes the lines will not exceed the limit. Constraint 30 tracks the state of charge of the bus to ensure that it is always between the safe level and the maximum state of charge, where $\sigma$ is the proportion of safe energy level to the maximum state of charge. Constraint 31 ensures that buses are fully charged when departing the depot for the first time. Constraint 32 ensures that the number of buses departing from each depot will not exceed its limit, where $\varepsilon^{\beta}$ is the parking limit of the depot. Finally, constraints 33 and 34 define the decision variables.

\subsection{Solving the problem}

Guedes and Borenstein [20] demonstrated that MDMVTVSP is an NP-hard problem. Since the problem studied in this paper is an extension of the MDMVTVSP considering the driving range limit and recharging demand, it is also NP-hard. In order to improve the potential of the methodology in practical application, an Adaptive Large Neighbourhood Search Algorithm (ALNS) is employed to solve the problem. This algorithm iteratively selects a pair of destroy and repair operators to optimise the solution, and the selection probability of each operator varies according to how much the solution is improved in the previous iteration. With this mechanism, the algorithm can select operators that are more suitable for the problem during the iterative process, and it can achieve a high-quality solution in a reasonable time frame. Besides, a local search procedure is implemented to enhance the efficiency of the algorithm. A flowchart illustrating the proposed algorithm is shown in Figure 3, and the steps of the algorithm are described below.

\section{Initialisation}

The initial solution is generated by a greedy construction algorithm similar to Adler [21]. First, all service trips are sorted according to their starting time and then iteratively inserted into the solution. Each inserted trip is first assigned to the schedule of the existing buses. The number of line changes is counted, and buses with less than the maximum number of line changes are allowed to insert trips at this stage. The algorithm calculates the cost of the trip according to its schedule. If the bus is unable to complete the schedule due to insufficient state of charge, the algorithm tries to insert a recharging trip into the schedule. For the bus that still cannot complete the schedule after the recharging trip is introduced, the algorithm changes its bus type to one with a larger battery or lower energy consumption rate. The increase of the cost during the whole process is counted in the insertion cost of the trip. The algorithm assigns the trip to the bus with the lowest insertion cost. If none

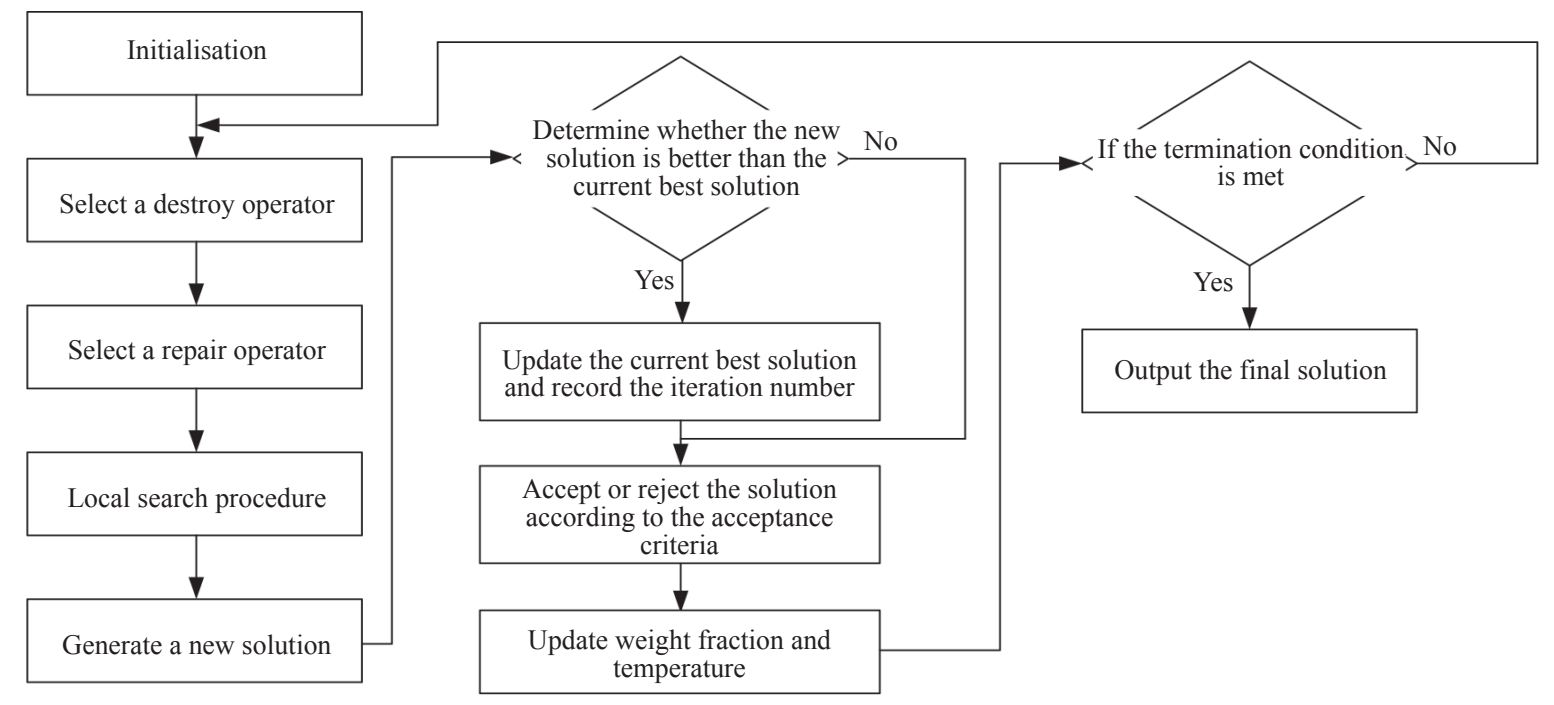

Figure 3 - Flow chart of the algorithm 
of the existing buses can perform the trip, the algorithm creates a new bus with the lowest cost for the trip. The algorithm stops until all the service trips are served.

\section{Destroy operators}

The destroy operators remove a series of trips from the solution with certain rules. Three kinds of operators are employed in the ALNS.

The RandomRemoval randomly removes a specified number of trips from the solution. The TimeRelatedRemoval aims to remove the series of trips with the closest start time. It first removes a randomly chosen trip and then repeatedly removes trips that have the closest start time to any of the removed trips. The NeighbourRemoval removes the series of trips most likely to be performed by the same bus. It starts with a randomly chosen trip and then removes the neighbouring trip closest to its end time, including $\xi_{n}$ trips closest to the start time of the neighbouring trip.

In each iteration, one of the operators removes $\delta$ trips from the solution, where $\delta$ is a random number between $\left[\xi_{\text {min }}, \xi_{\max }\right]$. After that, the algorithm searches for schedules with fewer than two trips and removes them. This process may increase the running time, but it can effectively improve the ability to reduce the fleet size.

\section{Repair operators}

When the solution is destroyed, the repair operators are employed to rebuild it. The regret insertion approach is employed in the ALNS. The basic greedy algorithm tends to insert the trip with the greatest increase in the operation cost later in the process, leaving only a few possibilities of searching the neighbourhood of the current solution. To avoid this behaviour, the regret insertion approach uses a regret-value which represents the expected costs of inserting a trip not in this operation but in a future operation.

For a trip $i$ to be inserted, the insertion costs and positions of all possible buses are calculated iteratively. If the insertion leads to an infeasible solution that violates the timetable, the insertion cost is set as a sufficiently large positive number $M$. If the inserted trip is feasible in timing but leads to infeasible energy requirements, the recharging trips are inserted into the schedule. Similar to the approach in the initialisation process, if the bus cannot complete the schedule after the recharging trip is inserted, the algorithm will try to complete the service by changing the bus type. The additional purchase cost brought by the type change will be added to the insertion cost of the trip. Let $j \in J$ be the index of the buses and $c_{i}^{j}$ be the insertion cost of bus $j$, where $J$ is sorted based on $c_{i}^{j}$. The regret value of the trip $i$ can be calculated according to equation 35, where $k$ is the calculation parameter of the regret value. In each iteration, the regret-k algorithm will insert the trip with the largest regret value. The algorithm will iterate until all trips are inserted into the solution. The regret-2, regret-3, and regret-4 algorithms are used in the ALNS.

regret $-\operatorname{trip}(i)=\sum_{j=1}^{k}\left(c_{i}^{j}-c_{i}^{1}\right)$

\section{Local search}

Due to the multi-depot and multi-vehicle type conditions investigated in this study, a local search procedure is introduced into the algorithm to enhance the search capability. The local search explores the alternative bus type and departure depot to achieve a lower operation cost. Let $u \in K, v \in D$, $(u, v)$ represent the bus type and depot information combination. The algorithm explores all the possible combinations while keeping the bus schedule unchanged. The combination leading to the minimal bus cost will be selected. After processing all the buses, due to the parking limit of depots, redundant buses must be moved from any depot where the number of parked buses exceeds the spare space of the depot. Similar to the approach of the regret insertion, the regret value of each bus in the depot that exceeds the limit is calculated to determine which bus is to be moved. Let $n \in N$ be the index of the buses and $c_{(u, v)}^{i n}$ be the total cost of bus $i$, where $N$ is sorted based on $c_{(u, v)}^{i n}$. The regret value of bus $i$ can be calculated according to equation 36 . Buses with the smaller regret values will move to other depots first.

$$
\begin{gathered}
\text { regret-vehicle }(i)=\sum_{n=1}^{k}\left(c_{(u, v)}^{i n}-c_{(u, v)}^{i 1}\right), \\
\forall u, u^{\prime} \in K, \forall v, v^{\prime} \in D, v \neq v^{\prime}
\end{gathered}
$$

\section{Acceptance and termination criteria}

A simulated annealing approach is employed to handle the acceptance of the solution. Let $s$ and $s^{\prime}$ be the solutions before and after the current iteration, respectively. The solution generated in each iteration is accepted with a probability $e^{\left(f(s)-f\left(\mathrm{~s}^{\prime}\right) / T\right)}$, where $T$ is the current temperature initialised by $T_{0}$ and decreases with the cooling rate $\alpha$, and $f(s)$ is the objective value of the solution $s$. 
Zhang A, Li T, Tu R, et al. The Effect of Nonlinear Charging Function and Line Change Constraints on Electric Bus Scheduling

Two termination conditions are applied in the algorithm, the algorithm terminates when $\eta_{\max }$ iterations have been executed, or $\eta_{\operatorname{maxNoimprove}}$ iterations occur without an improvement.

\section{RESULT ANALYSIS}

\subsection{Parameter values}

In this section, a case study is conducted based on a real-world transit network in Nanjing, China. The network has three bus lines and two depots, as shown in Figure 4. Both depots have a number of parking spaces and are equipped with charging infrastructures for recharging the buses. Depot 1 is a smaller depot at the terminal station that can serve 30 buses, while depot 2 is a large transit centre that can provide parking and recharging services for more than 100 buses. The three bus lines serve in total 518 trips, and the operation data of all lines (including the trip start time, duration, and end time) are extracted from the automatic vehicle locating (AVL) system. The information and charging

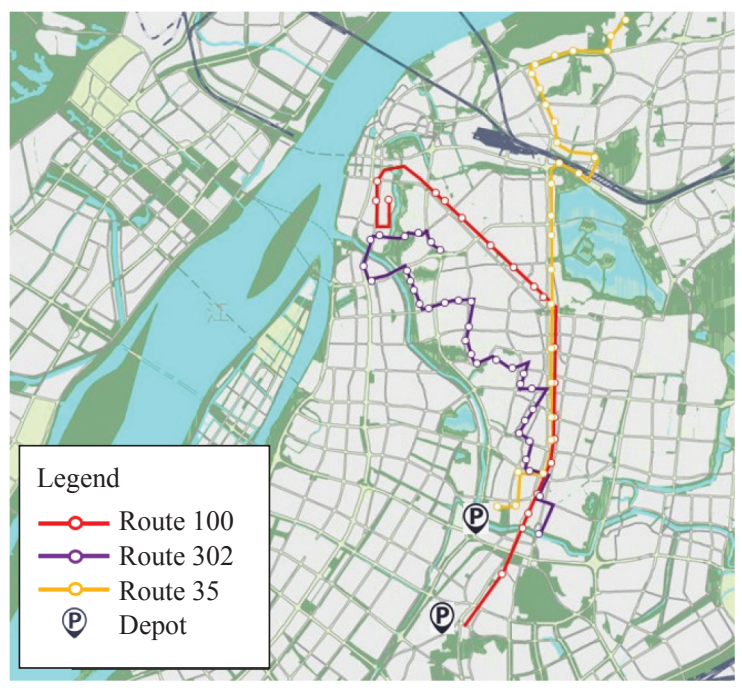

Figure 4 - Transit network in Nanjing
Table 1 - Parameters of electric bus types

\begin{tabular}{||c|c|c|c||}
\hline & Type A & Type B & Type C \\
\hline \hline $\begin{array}{c}\text { Battery capacity } \\
\text { [kWh] }\end{array}$ & 72 & 240 & 400 \\
\hline $\begin{array}{c}\text { Purchase cost } \\
\text { (CNY¥) }\end{array}$ & $2,450,000$ & $2,835,000$ & $3,150,000$ \\
\hline
\end{tabular}

functions of the three types of electric buses used in the case is extracted from [17], and it is detailed in Table 1. The piecewise linear approximation approach is used to model the charging functions of the buses. For the maintenance of the battery, the state of charge of the bus is required to be higher than $20 \%$ of the battery capacity during operation. In addition, the energy consumption rate of the bus during a deadhead trip is set as $1.5 \mathrm{kWh} / \mathrm{km}$, and the energy consumption rate in service is set as $1.8 \mathrm{kWh}$ $/ \mathrm{km}$ [17]. The electricity consumption cost $c_{e}$ is 0.07 yuan $/ \mathrm{kWh}$ [22], and the time-dependent $\operatorname{cost} c_{t}$ is 3 yuan/min [11]. There are 360 operating days per year, and the average life of the electric buses is calculated as 7.8 years [23].

Through the preliminary experimental analysis, parameters of ALNS are set as follows: the maximum iterations $\eta_{\max }$ is 2000 and $\eta_{\max \text { Noimprove }}$ is 500; the initial temperature $T_{0}$ is 50 and the cooling rate $\alpha$ is 0.99 . The number of trips deleted by the destroy operators in each iteration $\left[\xi_{\min }, \xi_{\max }\right]$ is set to $10-20$, and $\xi_{n}$ is 2 .

\subsection{Comparison between exact approach (CPLEX) and heuristic approach (ALNS)}

A small computational experiment is conducted to compare the results of the ALNS algorithm with the CPLEX solver. The instances are generated by randomly selecting a certain number of trips from all the timetable trips. The results are listed in Table 2.

Table 2 - Comparison between exact approach (CPLEX) and heuristic approach (ALNS)

\begin{tabular}{|c|c|c|c|c|c|c|c|}
\hline \multirow{2}{*}{$\begin{array}{l}\text { Number } \\
\text { of trips }\end{array}$} & \multicolumn{3}{|c|}{ CPLEX } & \multicolumn{3}{|c|}{ ALNS } & \multirow[b]{2}{*}{ Gap [\%] } \\
\hline & $\begin{array}{c}\text { Obj } \\
\text { (million yuan) }\end{array}$ & Mix & Time $[\mathrm{s}]$ & $\begin{array}{c}\text { Obj } \\
\text { (million yuan) }\end{array}$ & Mix & Time $[\mathrm{s}]$ & \\
\hline 20 & 42.24 & $\mathrm{~A}^{2} \mathrm{~B}^{6}$ & 4.5 & 42.24 & $\mathrm{~A}^{2} \mathrm{~B}^{6}$ & 52.6 & 0 \\
\hline 20 & 32.98 & $\mathrm{~A}^{2} \mathrm{~B}^{3}$ & 5.8 & 32.98 & $\mathrm{~A}^{2} \mathrm{~B}^{3}$ & 50.6 & 0 \\
\hline 25 & 49.98 & $\mathrm{~A}^{2} \mathrm{~B}^{7}$ & 8.7 & 49.98 & $\mathrm{~A}^{2} \mathrm{~B}^{7}$ & 90.7 & 0 \\
\hline 25 & 42.17 & $\mathrm{~A}^{2} \mathrm{~B}^{4}$ & 6.4 & 42.18 & $\mathrm{~A}^{2} \mathrm{~B}^{4}$ & 63.7 & 0.02 \\
\hline 30 & 51.38 & $\mathrm{~B}^{6}$ & $>3600$ & 51.38 & $\mathrm{~B}^{6}$ & 76.5 & - \\
\hline 30 & 47.82 & $\mathrm{~A}^{1} \mathrm{~B}^{5}$ & 114.1 & 47.82 & $\mathrm{~A}^{1} \mathrm{~B}^{5}$ & 85.8 & 0 \\
\hline
\end{tabular}


The maximum running time for CPLEX is set to 1 hour. The results show that although CPLEX takes less time to solve most of the instances, its solving time increases rapidly with the increase of number of trips in instances. In contrast, ALNS can provide high-quality solutions for all instances, and the running time is relatively stable. The advantage can be further enhanced when the number of trips increases.

\subsection{Comparisons of nonlinear and linear charging functions}

To compare the difference between using nonlinear charging function and linear charging function in the scheduling process, we solve the problem with different charging functions respectively. The Piecewise Linear (PL) functions represent the piecewise linear approximation of the charging functions referenced from [17]. Two linear approximation functions, shown in Figure 1 (LC1 and LC2), are used for comparison. The results for homogeneous and heterogeneous fleets are both calculated, and all results are the average of five random runs. To eliminate the effect of different line change constraints, the number of line changes is set to be large enough to allow buses to perform a flexible schedule.

The results are listed in Table 3. The first column indicates the bus type and charging function used in the scenario. Fleet size represents the number of buses required in the solution. The total cost of the solution is listed in the third column. Mix represents the number of buses required for each bus type in the solution. Recharging trips represent the total number of recharging trips of all buses in the daytime operation. After using the linear approximation function to solve the problem, we use the
PL function to check the schedule of each vehicle. The infeasible schedule represents the proportion of all bus schedules that are infeasible due to insufficient energy storage. Since the battery capacity of bus type A is small, it is infeasible to perform all trips when only bus type A is used. When LC1 approximation is adopted, due to the fast charging rate of LC1 approximation, buses are assumed to obtain more energy within the same charging time, enabling it to perform more trips. Therefore, it can be seen from the table that for solutions with heterogeneous fleets or homogeneous fleets of bus type $\mathrm{B}$, the fleet size and total cost in LC1 approximation solutions are both lower than those of the other solutions. However, due to the large deviation between the charging rate of LC1 approximation and PL function, a large proportion of bus schedules are infeasible, which means that buses will break down due to insufficient energy during the service. When only bus type $\mathrm{C}$ is used, fleet size and total cost do not show a significant difference between LC1 approximation solution and other solutions. This can be explained by the large battery capacity of the bus type $\mathrm{C}$, which significantly reduces the recharging trips compared with the other solutions. It also reduces the impact of different charging functions on the bus scheduling.

When LC2 approximation is adopted, the recharging trips of the solutions with both heterogeneous fleets and homogeneous fleets are higher than those with the PL function. The charging rate of LC2 approximation is lower than that of PL function between $0-80 \%$ state of charge, which means that the charging time at this stage will be longer when using LC2 approximation, and more buses are needed to fill the gap when other buses are charging.

Table 3 - Results of solutions with different charging functions

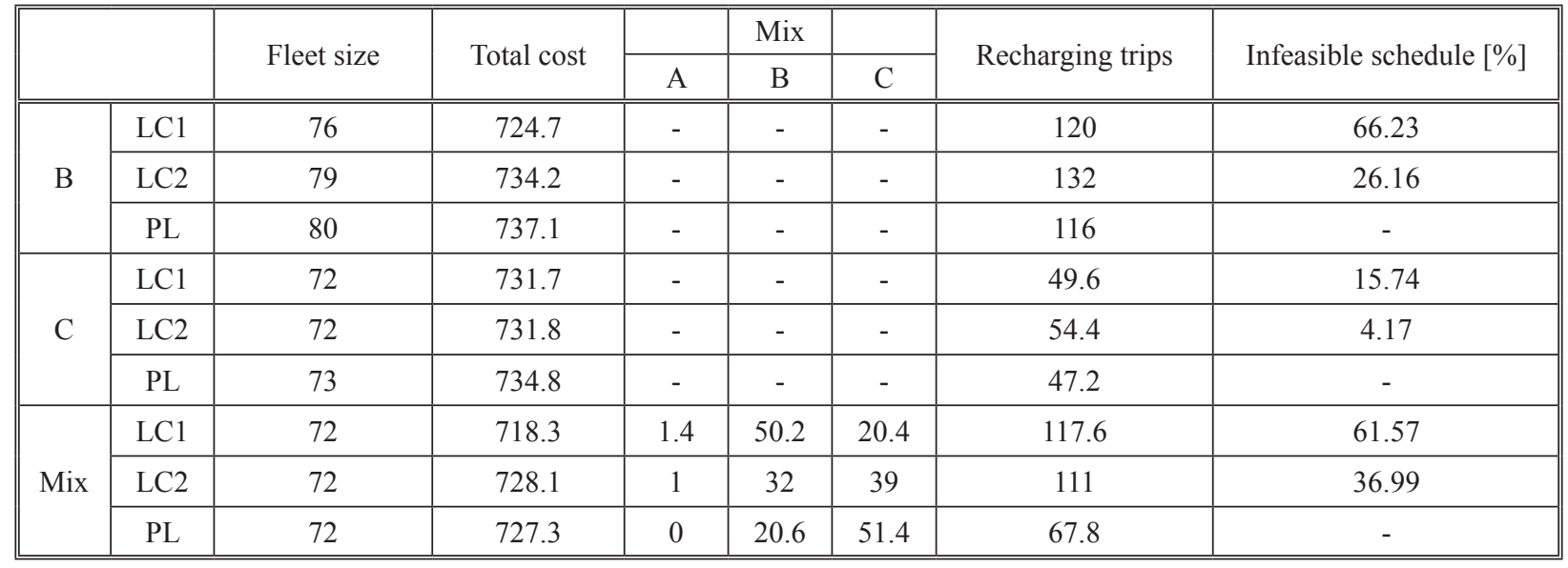


In general, conservative methods such as LC2 are preferred when linear approximation functions are used to approximate the real-world charging functions. It can be seen from Figure 1 that the curve of LC2 approximation is below the curve of PL and the real-world charging functions. Therefore, it is intuitively believed that although LC2 may conservatively estimate the charging speed, it ensures the feasibility of bus schedules. However, a certain proportion of bus schedules is still not feasible based on the result of this study. This is because the charging rate of LC2 approximation is higher than NL function at $80-100 \%$ state of charge. When the bus is recharged in this state of charge interval, the estimated charging time based on LC2 approximation for the same energy amount is shorter than the estimated time of PL function, which can result in insufficient energy charged by the bus within the estimated charging time.

In addition, it can be found that the proportion of infeasible bus schedules in the solutions with only bus type $\mathrm{C}$ are significantly lower than the solutions with only bus type B. Especially in the LC2 approximation solution with homogeneous fleets of type $\mathrm{C}$, only $4.17 \%$ of the bus schedules are infeasible. This indicates that the linear approximation approach is more suitable to estimate the charging rate for buses with larger batteries.

\subsection{Sensitive analysis of maximum number of lines changes}

To analyse the influence of different line change number constraints on scheduling, a sensitive analysis is conducted by setting four different values for the maximum line change numbers of a bus. According to the preliminary computational experiments, when this value is greater than or equal to 4 , the re- sult obtained will not change significantly. Therefore, we set the maximum value of this value to 4 . For each scenario, five random runs are performed, and average results are reported in Table 4. The fleet size and the total cost are the highest when $\theta=0$ (which means that the bus can only operate on a single line). When $\theta$ equals 1 , the total cost has a significant drop. As the value of $\theta$ varies from 1-4, the flexibility of the schedule is improved, thus reducing the total cost and fleet size. The comparison of the number of lines changes of different scenarios is interesting. Because of the line change constraints, the average of line changes of five runs when $\theta=1$ is only 38 . Although it increases with the relaxation of the constraints, there is no difference in the average number of line changes when $\theta$ increases from 2 to 4 . This indicates that it is more cost-effective to allow more line change operations for a small number of buses than to increase the number of line changes for all buses. In terms of the fleet composition, when buses are restricted to operate on a single line, the solution tends to use more buses with smaller batteries. When multiple shifts among different lines are allowed, more buses with a larger battery are used. The number of recharging trips in the solution also decreases due to the increasing number larger battery buses, which can perform more trips after fully charged. It indicates that when the flexibility of scheduling increases, buses with larger batteries are more efficient and thus preferred. On the other hand, although more recharging trips are required when each bus only serves one line, since the buses do not need deadhead trips between two terminal stations to serve trips of other lines, their deadhead mileages are shorter, which also reduces the electricity consumption.

Comparing these solutions, it can be observed that the largest drop of the total cost occurs when $\theta$ increases from 0 to 1 , while the deadhead mileage and

Table 4 - Results of solutions with different maximum number of lines changes

\begin{tabular}{|c|c|c|c|c|c|}
\hline & & $\theta=0$ & $\theta=1$ & $\theta=2$ & $\theta=4$ \\
\hline \multicolumn{2}{|c|}{ Fleet size } & 77 & 74 & 73 & 72 \\
\hline \multicolumn{2}{|c|}{ Total cost (million CNY) } & 734.2 & 730.4 & 728.3 & 727.3 \\
\hline \multirow{3}{*}{ Mix } & A & 2 & 1.2 & 0.2 & 0 \\
\hline & B & 44.2 & 28.4 & 27 & 20.6 \\
\hline & $\mathrm{C}$ & 30.8 & 44.4 & 45.8 & 51.4 \\
\hline \multicolumn{2}{|c|}{ Recharging trips } & 88.2 & 73.8 & 71.8 & 67.8 \\
\hline \multicolumn{2}{|c|}{ Number of line changes } & 0 & 38 & 66.2 & 66.2 \\
\hline \multicolumn{2}{|c|}{ Total deadhead mileage $[\mathrm{km}]$} & 461.2 & 495.3 & 504.2 & 507.7 \\
\hline \multicolumn{2}{|c|}{ Electricity consumption [kWh] } & 29592.4 & 29643.4 & 29656.8 & 29661.8 \\
\hline
\end{tabular}


the total number of line changes stay at a low level when $\theta$ equals 1 . Therefore, it is a very cost-effective solution, especially for bus companies that want to reduce the total cost while maintaining a low number of line changes. After that, when $\theta$ changes from 1 to 4 , the minimum cost keeps stable with more additional recharging trips and deadhead mileages. The result of the sensitivity analysis suggests that the line change constraints can be relaxed in certain circumstances, and this strategy can achieve lower costs in the process of public transit planning and operation.

\section{CONCLUSION}

In this paper, a multi-depot and multi-vehicle type electric vehicle scheduling problem with a nonlinear charging function is formulated. A piecewise linear approximation approach is used as the realistic charging function for a more feasible bus schedule. A constraint to restrict the number of bus line changes is introduced into the model to reduce the delays and management difficulties caused by the large number of the line change in large transit networks. The objective of the model aims to minimise the total cost of the network, which consists of the fleet acquisition cost and the operation cost. In order to efficiently solve this comprehensive problem, an improved ALNS algorithm is proposed.

The optimisation problem proposed in this paper is implemented to a case study with a real transit network dataset. The results with linear and piecewise linear approximation charging functions are compared. Based on the nonlinear charging function of the buses, two different linear approximations are adopted. The results show that using either aggressive or conservative linear approximation can lead to a significant proportion of infeasible bus schedules, leading to unexpected break-down during the bus service associated with additional emergency treatment costs. Besides, a sensitivity analysis is performed to assess the influence of different maximum number of line changes in the schedule. The results show that both the fleet size and the total cost decrease gradually as the maximum number of line changes increases, proving that the operation cost can be reduced when more flexible operating strategies are allowed. With the approach proposed in this paper, the bus company can reach a balance between reducing costs and controlling the number of line changes.

Future studies can be conducted based on this work. In the bus scheduling problem, besides the vehicle scheduling, the crew scheduling can be integrated for a better and more efficient management. In addition, the uncertainty of the bus energy consumption can be considered for a more realistic energy consumption estimation, rather than a constant energy consumption rate adopted in this paper.

\section{ACKNOWLEDGEMENT}

The research in this paper was jointly supported by the National Natural Science Foundation of China (No. 41877395), the EU-funded project MODALES (grant agreement No 815189), and the China Postdoctoral Science Foundation (No.2020M681466).

张爱佳, 李铁柱, 涂然, 董长印, 陈海波, 高建兵, 刘野

非线性充电函数和车辆跨线路约束对纯电动公 交车辆调度的影响

摘要

充电计划是纯电动公交调度方案的关键部分。由 于电动汽车的实际充电函数为非线性, 因此准确地 估算充电时间非常困难。为了研究考虑非线性充电 函数时的电动公交调度问题, 本文提出了一种混合 整数规划下的分段线性近似方法以研究多车场多车 型条件下的电动车辆调度问题。本文的数学模型目 标在于最小化包括车辆购置成本和运营成本的总调 度成本。同时基于现实的角度考虑, 模型增加了对 车辆的跨线路次数的约束。为了高效地求解问题, 本文提出了一种改进的启发式算法。最后, 本文使 用了真实数据用于案例研究。结果表明线性近似充 电函数与分段线性近似充电函数所计算出的结果存 在较大偏差，这表明采用分段线性近似的充电函数 能够避免得出不可行的调度方案。此外, 结果也显 示了所提出的车辆跨线路次数约束可以成为公交公 司的一种有效的调度策略。

\section{关键词}

电动车辆调度问题; 非线性充电函数; 跨线路 次数约束; 混合整数规划; 启发式算法。

\section{REFERENCES}

[1] Li J-Q. Battery-electric transit bus developments and operations: A review. International Journal of Sustainable Transportation. 2016;10(3): 157-69. DOI: 10.1080/15568318.2013.872737

[2] Liu T, Ceder AA. Battery-electric transit vehicle scheduling with optimal number of stationary chargers. Transportation Research Part C: Emerging Technologies. 2020;114:118-39. DOI: 10.1016/j.trc.2020.02.009

[3] Bertossi AA, Carraresi P, Gallo G. On some matching problems arising in vehicle scheduling models. Networks. 1987;17(3): 271-81. DOI: 10.1002/net.3230170303

[4] Bunte S, Kliewer N. An overview on vehicle schedul- 
ing models. Public Transport. 2009;1(4): 299-317. DOI: 10.1007/s12469-010-0018-5

[5] Kliewer N, Mellouli T, Suhl L. A time-space network based exact optimization model for multi-depot bus scheduling. European journal of operational research. 2006;175(3): 1616-27. DOI: 10.1016/j.ejor.2005.02.030

[6] Kliewer N, Gintner V, Suhl L. Line change considerations within a time-space network based multi-depot bus scheduling model. In: Hickman M, Mirchandani P, Voß S. (eds) Computer-aided Systems in Public Transport. Springer; 2008. p. 57-70. DOI: 10.1007/978-3-54073312-6_4

[7] Chao Z, Xiaohong C. Optimizing battery electric bus transit vehicle scheduling with battery exchanging: Model and case study. Procedia-Social and Behavioral Sciences. 2013;96: 2725-36. DOI: 10.1016/j.sbspro.2013.08.306

[8] Li J-Q. Transit bus scheduling with limited energy. Transportation Science. 2014;48(4): 521-39. DOI: $10.1287 /$ trsc.2013.0468

[9] Wen $\mathrm{M}$, et al. An adaptive large neighborhood search heuristic for the electric vehicle scheduling problem. Computers \& Operations Research. 2016;76: 73-83. DOI: 10.1016/j.cor.2016.06.013

[10] Tang X, Lin X, He F. Robust scheduling strategies of electric buses under stochastic traffic conditions. Transportation Research Part C: Emerging Technologies. 2019;105: 163-82. DOI: 10.1016/j.trc.2019.05.032

[11] Rogge M, van der Hurk E, Larsen A, Sauer DU. Electric bus fleet size and mix problem with optimization of charging infrastructure. Applied Energy. 2018;211: 28295. DOI: 10.1016/j.apenergy.2017.11.051

[12] Li L, Lo HK, Xiao F. Mixed bus fleet scheduling under range and refueling constraints. Transportation Research Part C: Emerging Technologies. 2019;104: 443-62. DOI: 10.1016/j.trc.2019.05.009

[13] Rinaldi M, Picarelli E, D'Ariano A, Viti F. Mixed-fleet single-terminal bus scheduling problem: Modelling, solution scheme and potential applications. Omega. 2020;96: 102070. DOI: 10.1016/j.omega.2019.05.006

[14] Pelletier S, Jabali O, Laporte G, Veneroni M. Battery degradation and behaviour for electric vehicles: Review and numerical analyses of several models. Transportation Research Part B: Methodological. 2017;103: 15887. DOI: $10.1016 /$ j.trb.2017.01.020

[15] Montoya A, Guéret C, Mendoza JE, Villegas JG. The electric vehicle routing problem with nonlinear charging function. Transportation Research Part B: Methodological. 2017;103: 87-110. DOI: 10.1016/j.trb.2017.02.004

[16] Froger A, Mendoza JE, Jabali O, Laporte G. Improved formulations and algorithmic components for the electric vehicle routing problem with nonlinear charging functions. Computers \& Operations Research. 2019;104: 256-94. DOI: 10.1016/j.cor.2018.12.013

[17] Olsen N, Kliewer N. Scheduling Electric Buses in Public Transport: Modeling of the Charging Process and Analysis of Assumptions. Logist Res. 2020;13(1): 4. DOI: 10.23773/2020_4

[18] Hõimoja H, Rufer A, Dziechciaruk G, Vezzini A, editors. An ultrafast EV charging station demonstrator. International Symposium on Power Electronics Power Electronics, Electrical Drives, Automation and Motion, 20-22 June 2012, Sorrento, Italy. IEEE; 2012. p. 1390-5. DOI: 10.1109/SPEEDAM.2012.6264617

[19] Zuo X, et al. A new formulation of the electric vehicle routing problem with time windows considering concave nonlinear charging function. Journal of Cleaner Production. 2019;236: 117687. DOI: 10.1016/j.jclepro.2019.117687

[20] Guedes PC, Borenstein D. Column generation based heuristic framework for the multiple-depot vehicle type scheduling problem. Computers \& Industrial Engineering. 2015;90: 361-70. DOI: 10.1016/j.cie.2015.10.004

[21] Adler JD. Routing and scheduling of electric and alternative-fuel vehicles. Arizona State University; 2014.

[22] Yao E, Liu T, Lu T, Yang Y. Optimization of electric vehicle scheduling with multiple vehicle types in public transport. Sustainable Cities and Society. 2020;52: 101862. DOI: $10.1016 / j . s c s .2019 .101862$

[23] Wang Y, Huang Y, Xu J, Barclay N. Optimal recharging scheduling for urban electric buses: A case study in Davis. Transportation Research Part E: Logistics and Transportation Review. 2017;100: 115-32. DOI: 10.1016/j.tre.2017.01.001 